\title{
Anti-inflammatory effect of ethanolic extract from Myagropsis myagroides on murine macrophages and mouse ear edema
}

\author{
Eun-Ji Joung ${ }^{1}$, Min-Sup Lee ${ }^{1}$, Ji-Woong Choi ${ }^{1}$, Jong-Soon Kim ${ }^{1}$, Taisun Shin ${ }^{2}$, Bok-Mi Jung ${ }^{2}$, Na Young Yoon ${ }^{3}$, \\ Chi-Won Lim³, Jae-II Kim and Hyeung-Rak Kim ${ }^{1 *}$
}

\begin{abstract}
Background: This study aims to investigate anti-inflammatory effect of ethanolic extract of Myagropsis myagroides (EMM) in the lipopolysaccharide (LPS)-stimulated RAW 264.7 macrophages and the phorbol 12-myristate 13-acetate (PMA)-induced ear edema in mice, and to clarify its underlying molecular mechanisms.

Methods: The levels of nitric oxide (NO), prostaglandin $E_{2}\left(P_{G E}\right)$, and pro-inflammatory cytokines were measured by Griess assay and enzyme linked immunosorbent assay. The expressions of inducible nitric oxide synthase (iNOS), cyclooxygenase-2 (COX-2), mitogen-activated protein kinases (MAPKs), and Akt were measured using Western blotting. Nuclear translocation and transcriptional activation of nuclear factor-kB (NF-kB) were determined by immunocytochemistry and reporter gene assay, respectively. PMA-induced mouse ear edema was used as the animal model of inflammation. Anti-inflammatory compounds in EMM were isolated using high-performance liquid chromatography and identified by nuclear magnetic resonance.

Results: EMM significantly inhibited the production of $\mathrm{NO}, \mathrm{PGE}_{2}$, and pro-inflammatory cytokines in a dose-dependent manner and suppressed the expression of iNOS and COX-2 in LPS-stimulated RAW 264.7 cells. EMM strongly suppressed nuclear translocation of NF-KB by preventing degradation of inhibitor of $\mathrm{KB}-\mathrm{a}$ as well as by inhibiting phosphorylation of Akt and MAPKs. EMM reduced ear edema in PMA-induced mice. One of the anti-inflammatory compounds in EMM was identified as 6,6'-bieckol.
\end{abstract}

Conclusions: These results suggest that the anti-inflammatory properties of EMM are associated with the down-regulation of iNOS, COX-2, and pro-inflammatory cytokines through the inhibition of NF-kB pathway in LPS-stimulated macrophages.

Keywords: Anti-inflammation, Myagropsis myagroides, MAPK, NF-kB, RAW 264.7 cells

\section{Background}

Inflammation is a complex response of host defense against microbial infection, endotoxin exposure, or cell injury, and ultimately leads to the restoration of normal cell structure and function. Macrophages are known to play a key role in host defense mechanism, and are activated by exposure to interferon- $\gamma$, pro-inflammatory cytokines, and bacterial lipopolysaccharides (LPSs) [1,2].

\footnotetext{
* Correspondence: hrkim@pknu.ac.kr

'Department of Food Science and Nutrition, Pukyong National University, Busan 608-737, South Korea

Full list of author information is available at the end of the article
}

Activated macrophages play a pivotal role in inflammatory diseases by excessive production of proinflammatory cytokines, including tumor necrosis factor (TNF)- $\alpha$, interleukin (IL)-1 $\beta$, and IL-6, and inflammatory mediators such as nitric oxide (NO) and prostaglandin $\mathrm{E}_{2}\left(\mathrm{PGE}_{2}\right)[3,4]$. Expressions of these cytokines and mediators are regulated by the activation of nuclear factorkappaB (NF-kB), which is critically involved in the pathogenesis of rheumatism and other chronic inflammatory diseases [5]. In the unstimulated condition, NF- $k B$ is located in the cytoplasm as an inactive complex bound to inhibitor of kappaB and (IкB)- $\alpha$. Treatment of LPS activates the IKB- $\alpha$ kinase (IKK) complex, resulting 
in the phosphorylation, ubiquitination, and degradation of $I \kappa B-\alpha$, inducing the translocation of NF- $\kappa B$ into the nucleus for transcriptional activation. The activation of $\mathrm{NF}-\mathrm{kB}$ is also regulated by cellular kinases such as mitogen-activated protein kinases (MAPKs) and Akt $[6,7]$. Hence, substances which inhibit the activation of $\mathrm{NF}-\mathrm{\kappa} \mathrm{B}$ are considered as potential anti-inflammatory agents.

Myagropsis myagroides is one of the most abundant brown algae, which has been used as a part of the diet and folk medicines in Korea, Japan, and China for centuries. This species contains high level of phlorotannins and pigments. Recently, phlorotannins have been reported to have several biological activities, such as antioxidation [8], antidiabetic complications [9], antiamnesia [10], anti-inflammation [11], and hepatoprotection [12]. As a biological activity of M. myagroides, previous study revealed a cytoprotective effect on carbon tetrachloride-induced hepatotoxicity by its antioxidant properties [13]. Recently, fucoxanthin isolated from $M$. myagroides inhibited the production of inflammatory mediators and pro-inflammatory cytokines via inhibiting NF- $\kappa B$ activation in RAW 264.7 cells [14]. However, we found an anti-inflammatory activity in the ethanolic extract of sun-dried $M$. myagroides (EMM) in LPSstimulated RAW 264.7 cells. Since fucoxanthin prone to rapidly oxidized during sun-drying due to instability under oxygen and sun light exposure, we hypothesized that other anti-inflammatory compounds might be exist in EMM. Furthermore, we confirmed that EMM does not contain any detectable amount of fucoxanthin by HPLC chromatographic analysis at $410 \mathrm{~nm}$. To our knowledge, no previous study has been reported on the anti-inflammatory activity of $M$. myagroides except fucoxanthin. Therefore, we investigated the anti-inflammatory and anti-edema properties of EMM and its underlying mechanisms using cultured RAW 264.7 cells and identified antiinflammatory compound in EMM.

\section{Methods}

\section{Chemicals}

CellTiter96 ${ }^{\circledR}$ AQueous One Solution Cell Proliferation assay kit, luciferase assay kit, murine NF- $\mathrm{BB}$ promoter/ luciferase DNA, pRL-TK DNA and reverse transcriptase were obtained from Promega (Madison, WI, USA). Primary antibodies and secondary antibodies were purchased from Santa Cruz Biotechnology (Santa Cruz, CA, USA). Enhanced chemiluminescence (ECL) detection kit was obtained from GE Healthcare BioScience (Piscataway, NJ, USA). Dulbecco's modified Eagle's medium (DMEM), penicillin-streptomycin mixture, $\quad 0.25 \%$ trypsin-ethylenediaminetetraacetic acid (EDTA), and fetal bovine serum (FBS) were purchased from Gibco BRL Life Technologies (Grand Island, NY,
USA). 4,6-Diamidino-2-phenylindole (DAPI), Alexa Fluor $^{\circledR}$ 488-conjugated secondary antibody, Lipofectamine/Plus, and TRIzol were purchased from Invitrogen (Carlsbad, CA, USA). LPS (Escherichia coli O55: B5), bovine serum albumin (BSA), 2',7'-dichlorofluorescein diacetate (DCF-DA), dimethyl sulfoxide (DMSO), indomethacin (Indo), and phorbol 12-myristate 13acetate (PMA) were purchased from Sigma-Aldrich Corporation (St. Louis, MO, USA). Specific kinase inhibitors (Wortmannin, PD98059, SP600125, and SB203580) were purchased from Abcam (Cambridge, UK).

\section{Preparation of EMM}

M. myagroides was harvested from the coast of Busan, South Korea, in April 2010. Taxonomic identification was confirmed by an agal taxonomist (C.K Choi), at the Department of Ecological Engineering, Pukyong National University, Korea. The seaweed was rinsed in tap water to remove salt, and air-dried under sunshine for 3 days. A dried sample was ground with a hammer mill and the powder was stored at $-20^{\circ} \mathrm{C}$ until used. The dried powder $(500 \mathrm{~g})$ of $M$. myagroides was extracted three times with $96 \%$ ethanol ( $3 \mathrm{~L} /$ each) for $3 \mathrm{~h}$ at $70^{\circ} \mathrm{C}$. The combined extracts were filtered and concentrated using rotary vacuum evaporator (Tokyo Eyela, Tokyo, Japan) at $40^{\circ} \mathrm{C}$ and freeze-dried EMM (89.7 g) was stored in freezer $\left(-70^{\circ} \mathrm{C}\right)$ until used.

\section{Cell culture and viability assay}

Murine macrophage RAW 264.7 cells (ATCC, Rockville, MD, USA) were cultured at $37^{\circ} \mathrm{C}$ in DMEM supplemented with $10 \%$ FBS, penicillin (100 units $/ \mathrm{mL}$ ), and streptomycin sulfate $(100 \mu \mathrm{g} / \mathrm{mL})$. Cell viability was determined by 3-(4,5-dimethylthiazol-2-yl)-5-(3-carboxymethoxyphenyl)-2-(4-sulfophenyl)-2H-tetrazolium (MTS) assay using a CellTiter96 ${ }^{\circledR}$ AQ ueous One Solution Cell Proliferation assay kit according to the manufacturer's instruction. Briefly, cells were inoculated at a density of $5 \times$ $10^{5}$ cells/well into 96 -well plate and cultured at $37^{\circ} \mathrm{C}$ for $24 \mathrm{~h}$. The culture medium was replaced with $200 \mu \mathrm{L}$ of serial dilutions of EMM, and the cells incubated for 24 h. The medium was replaced with $95 \mu \mathrm{L}$ fresh medium and $5 \mu \mathrm{L}$ MTS solution. After $1 \mathrm{~h}$, the absorbance at 490 $\mathrm{nm}$ was measured using a microplate reader (Glomax Multi Detection System, Promega).

\section{Measurement of NO, PGE 2 , TNF- $a$, IL- $1 a$ and IL- 6}

RAW 264.7 cells were plated in a 12 -well plate at a density of $1 \times 10^{6}$ cells/well and incubated for $24 \mathrm{~h}$. Cultured cells were treated with various concentrations of EMM for $1 \mathrm{~h}$, and then stimulated with $1 \mu \mathrm{g} / \mathrm{mL}$ LPS for $24 \mathrm{~h}$. Cultured media were collected after centrifugation at $2,000 \mathrm{~g}$ for $10 \mathrm{~min}$ and stored at $-70^{\circ} \mathrm{C}$ until 
tested. The nitrite concentration in the cultured media was measured as an indicator of NO production, according to the Griess reaction [15]. Levels of $\mathrm{PGE}_{2}, \mathrm{IL}-1 \beta$, IL-6, and TNF- $\alpha$ in cultured media were quantitated by enzyme-linked immunosorbent assay (ELISA, R\&D Systems, Minneapolis, MN, USA) according to the manufacturer's instructions.

\section{Reverse transcription-polymerase chain reaction (RT-PCR)}

RAW 264.7 cells placed in a 6-well plate were pretreated with EMM for $1 \mathrm{~h}$ and then stimulated with LPS for $6 \mathrm{~h}$. Total RNA from each group was isolated with the TRIzol reagent. Five microgram of total RNA was used for reverse transcription using oligo-dT-adaptor primer and superscript reverse transcriptase. PCR was carried out with the gene-specific primers: COX-2 sense, 5'CAGCAAATCCTTGCTGTTCC-3'; COX-2 antisense, 5'-TGGGCAAAGAATGCAAACAT-3'; iNOS sense, 5'CACCTTGGAGTTCACCCAGT-3'; iNOS antisense, 5'-ACCACTCGTACTTGGGATGC-3'; glyceraldehyde 3-phosphate dehydrogenase (GAPDH) sense, 5'GACCCCTTCATTGACCTCAA-3'; GAPDH antisense, 5'-CTTCTCCATGGTGGTGAAGA-3'. GAPDH was used as an internal standard to evaluate relative expression of COX-2 and iNOS. Densitometric analysis of the data obtained from at least three independent experiments was performed using cooled CCD camera system EZ-Capture II and CS analyzer ver. 3.00 software (ATTO \& Rise Co., Tokyo, Japan).

\section{Transient transfection and luciferase assay}

Murine NF-kB promoter/luciferase DNA (1 $\mu \mathrm{g})$ along with 20 ng control pRL-TK DNA was transiently transfected into $2 \times 10^{5}$ RAW 264.7 cells/well in a 24-well plate using Lipofectamine/Plus reagents for $40 \mathrm{~h}$. Cells pretreated with $0-100 \mu \mathrm{g} / \mathrm{mL}$ EMM for $1 \mathrm{~h}$ were stimulated with LPS $(1 \mu \mathrm{g} / \mathrm{mL})$ for $6 \mathrm{~h}$. Each well was washed twice with cold phosphate-buffered saline (PBS), harvested in $100 \mu \mathrm{L}$ of lysis buffer (0.5 mM HEPES, pH 7.8, $1 \%$ Triton $\mathrm{N}-101,1 \mathrm{mM} \mathrm{CaCl}_{2}$, and $1 \mathrm{mM} \mathrm{MgCl} 2$ ) and used for the measurement of luciferase activity using a luciferase assay kit. Luminescence was measured on a TopCount microplate scintillation and luminescence counter (PerkinElmer, Santa Clara, CA, USA) in singlephoton counting mode for $0.1 \mathrm{~min} /$ well, following a 5 min adaptation in the dark. Luciferase activity was normalized to the expression of control pRL-TK.

\section{Preparation of cytosolic and nuclear extracts}

RAW 264.7 cells $\left(5 \times 10^{6}\right.$ cells/well $)$ pretreated with EMM for $1 \mathrm{~h}$ were stimulated with LPS for $0.5 \mathrm{~h}$. Cells were washed twice with cold PBS and harvested. Cell pellets were resuspended in $300 \mu \mathrm{L}$ of hypotonic buffer [10 mM HEPES/KOH, $10 \mathrm{mM} \mathrm{KCl,} 2 \mathrm{mM} \mathrm{MgCl}_{2}, 0.1$
mM EDTA, $1 \mathrm{mM}$ DTT, and $0.5 \mathrm{mM}$ phenylmethylsulfonyl fluoride (PMSF), $\mathrm{pH}$ 7.9] and incubated on ice for $15 \mathrm{~min}$. After vortexing for $10 \mathrm{~s}$, homogenates were separated into supernatants (cytoplasmic compartments) and pellets (nuclear components) by centrifugation at $13,000 \mathrm{~g}$ for $10 \mathrm{~min}$. The pellet was gently resuspended in $40 \mu \mathrm{L}$ complete lysis buffer $(50 \mathrm{mM} \mathrm{HEPES} / \mathrm{KOH}, 50$ $\mathrm{mM} \mathrm{KCl}, 1 \mathrm{mM}$ DTT, $300 \mathrm{mM} \mathrm{NaCl}, 1 \%$ IGEPAL CA630, $0.1 \mathrm{mM}$ EDTA, 10\% glycerol, and 0.5 mM PMSF, $\mathrm{pH} 7.9$ ) and centrifuged at $13,000 \mathrm{~g}$ for $20 \mathrm{~min}$ at $4^{\circ} \mathrm{C}$. The supernatant was used as the nuclear extract.

\section{Western immunoblot analysis}

RAW 264.7 cells were incubated with various concentrations of EMM for $1 \mathrm{~h}$ and stimulated with LPS $(1 \mu \mathrm{g} / \mathrm{mL})$ for $30 \mathrm{~min}$. RAW 264.7 cells were washed twice with cold PBS and lysed with lysis buffer (50 mM Tris- $\mathrm{HCl}, \mathrm{pH} 7.5$, $150 \mathrm{mM} \mathrm{NaCl}, 1 \%$ IGEPAL CA-630, 1\% Tween 20, 0.1\% SDS, $1 \mathrm{mM} \mathrm{Na} \mathrm{VO}_{4}, 10 \mu \mathrm{g} / \mathrm{mL}$ leupeptin, $50 \mathrm{mM} \mathrm{NaF}$, and $1 \mathrm{mM}$ PMSF) on ice for $1 \mathrm{~h}$. After centrifugation at $18,000 \mathrm{~g}$ for $10 \mathrm{~min}$, the protein concentrations in the supernatants were determined, and aliquots of the protein (40 $\mu \mathrm{g}$ ) were separated by sodium dodecyl sulfatepolyacrylamide gel electrophoresis (SDS-PAGE) and transferred onto a nitrocellulose membrane. The membrane was blocked with $5 \%$ nonfat dry milk in Trisbuffered saline with $0.1 \%$ Tween 20 (TBST) for $1 \mathrm{~h}$, followed by the incubation for $2 \mathrm{~h}$ with primary antibody in TBST containing 5\% nonfat dry milk. The blots were treated with horseradish peroxidase-conjugated secondary antibody in TBST containing 5\% nonfat dry milk for $1 \mathrm{~h}$, and immune complexes were detected using an ECL detection kit. Densitometric analysis of the data obtained from at least three independent experiments was performed using cooled CCD camera system EZ-Capture II and CS analyzer ver. 3.00 software.

\section{Immunofluorescence analysis}

RAW 264.7 cells were maintained on glass coverslips (SPL Lifesciences Co., Gyeonggi-do, Korea) to analyze nuclear localization of NF-kB in 24-well plates for $24 \mathrm{~h}$. Cells treated with EMM for $1 \mathrm{~h}$ were incubated with LPS $(1 \mu \mathrm{g} / \mathrm{mL})$ for $30 \mathrm{~min}$ as described in Lee et al. [16]. Cells were fixed in $4.0 \%$ paraformaldehyde in PBS for 15 min at room temperature, and then permeabilized with $0.5 \%$ Triton X-100 in PBS for 10 min. Cells were washed with PBS and blocked with 3\% BSA/PBS for $30 \mathrm{~min}$. Thereafter, cells were incubated with an anti-NF-kB polyclonal antibody diluted in 3\% BSA/PBS for $2 \mathrm{~h}$, and incubated with Alexa Fluor ${ }^{\circledR}$ 488-conjugated secondary antibody diluted in 3\% BSA/PBS for $1 \mathrm{~h}$. Cells were stained with $2 \mu \mathrm{g} / \mathrm{mL}$ DAPI and images were captured using an LSM700 laser scanning confocal microscope (Carl Zeiss, Oberkochen, Germany). 


\section{Mouse model and PMA-induced ear edema}

Animal studies were conducted after the experimental protocols and procedures were approved by the Animal Ethics Committee of the Pukyong National University. ICR mice (male, 25-30 g) were purchased from the Samtako Bio Korea Co. (Gyeonggido, Korea) and permitted free access to a standard chow diet and tap water. All mice were acclimatized for 1 week prior to the experiments and maintained at $22 \pm 2{ }^{\circ} \mathrm{C}$ with a relative humidity of $50 \pm 5 \%$ and $12 \mathrm{~h}$ light-dark cycle. Ear edema was induced on the right ear of mouse with PMA according to Garrido et al. [16]. Briefly, the control group received normal saline, and the other three groups include a PMA alone, PMA + Indo (a positive control), and EMM administered groups (PMA + EMM). The left ear (reference) received the vehicle (30 $\mu \mathrm{L}$ acetone). PMA (6 $\mu \mathrm{g} /$ ear in $30 \mu \mathrm{L}$ acetone) was applied to the inner surface of the right ear of ICR mouse. EMM $(90$ $\mu \mathrm{g}$ per ear in $30 \mu \mathrm{L}$ acetone) or Indo $(1 \mathrm{mg} /$ ear in $30 \mu \mathrm{L}$ acetone) was topically administered $1 \mathrm{~h}$ before PMA application. Six hours after PMA application, mice were killed by cervical dislocation and a $6 \mathrm{~mm}$ diameter disc from each ear was removed with a metal punch and weighed. Ear edema weight was calculated by subtracting the weight of the left ear from the right ear (treatment). Inhibition percentage (IP) was expressed as a reduction in weight compared to the PMA-treated group.

\section{Isolation of phlorotannins from EMM}

Aliquots of EMM were separated by Shimadzu highperformance liquid chromatography (HPLC) system with Luna RP-18 [Luna C18(2), $5 \mu \mathrm{m}, 250 \times 10 \mathrm{~mm}$, Phenomenex, Torrence, CA, USA]. The separation of EMM was conducted using the mobile phase of $0.1 \%$ formic acid in water (solvent $\mathrm{A}$ ) and $0.1 \%$ formic acid in acetonitrile (solvent B). The elution profile consisted of a linear gradient from 20 to $100 \%$ solvent B for $110 \mathrm{~min}$ and hold for $15 \mathrm{~min}$ and then re-equilibration of the column with $20 \%$ solvent $\mathrm{B}$ for $15 \mathrm{~min}$. The flow rate was 0.34 $\mathrm{mL} / \mathrm{min}$ at $35^{\circ} \mathrm{C}$ oven temperature and detection was performed at $270 \mathrm{~nm}$. The purity was determined by HPLC (purity: >99\%) and the chemical structure of the isolated compounds were identified as eckol [17] and 6,6'-bieckol $[8,12]$ by comparing their ${ }^{1} \mathrm{H}$ - and ${ }^{13} \mathrm{C}$-NMR data.

\section{Statistical analysis}

Data were expressed as the means \pm standard deviations (SDs) from at least three separate experiments unless
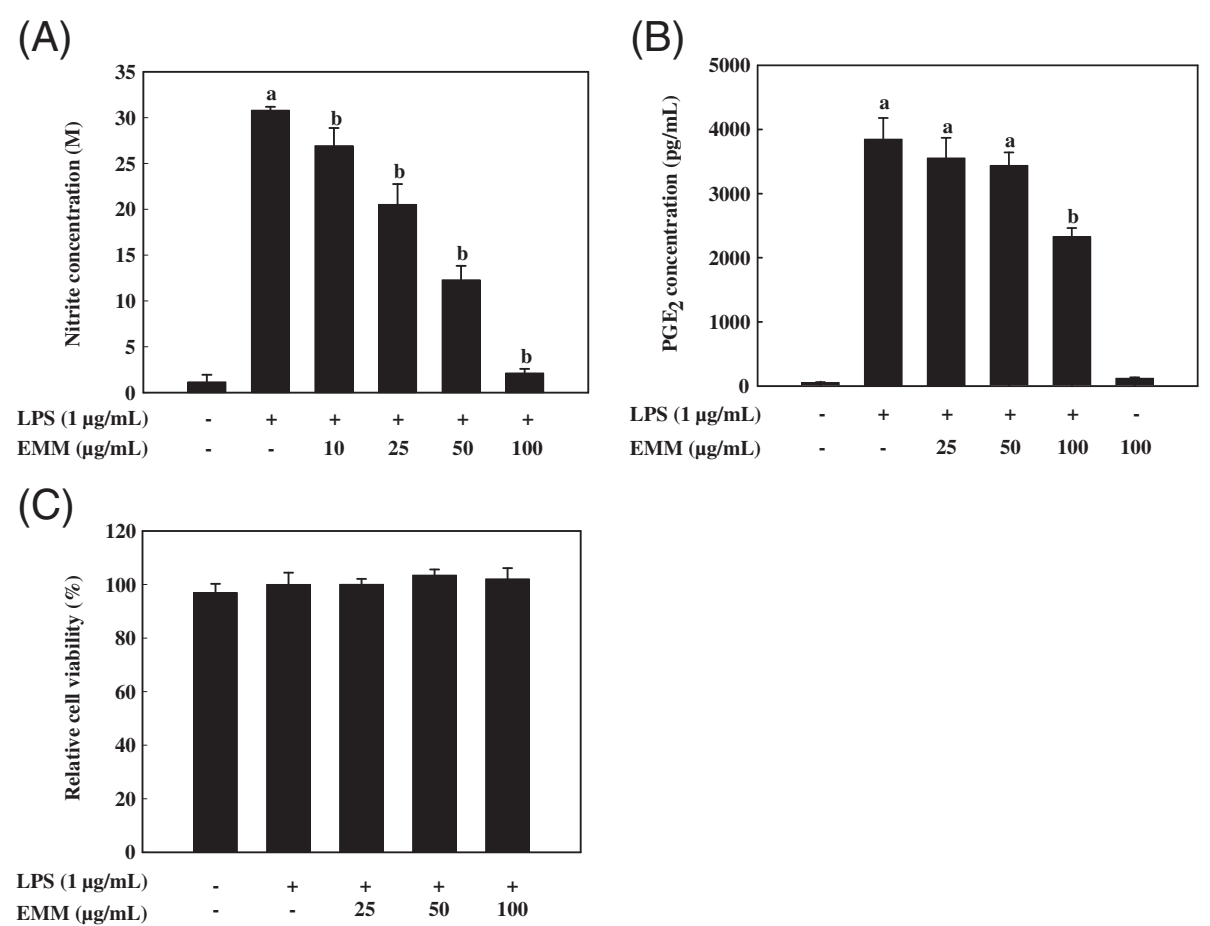

Figure 1 Effect of EMM on LPS-induced NO and PGE 2 production in RAW 264.7 cells. Cells pretreated with different concentrations of EMM for $1 \mathrm{~h}$ were stimulated with LPS $(1 \mu \mathrm{g} / \mathrm{mL})$ for $24 \mathrm{~h}$. The treated culture media were used to measure the amount of NO production (A) and $\mathrm{PGE}_{2}$ production (B). Cytotoxic effect of EMM was measured by MTS assay $(\mathbf{C})$. Values are the means \pm SDs of three independent experiments ${ }^{a} p<0.05$ indicates significant differences compared to the control group. ${ }^{b} p<0.05$ indicates significant differences compared to the LPS-only treated group. 
otherwise indicated. Data were analyzed using one-way analysis of variance. Differences were considered significant at values of $p<0.05$. All analyses were performed using SPSS for Windows, version 10.07 (SPSS Inc., Chicago, IL, USA).

\section{Results}

Effect of EMM on LPS-induced $\mathrm{NO}$ and $\mathrm{PGE}_{2}$ production

NO production, measured as nitrite, was increased by LPS treatment; however, EMM significantly reduced NO levels in LPS-stimulated cells in a dose-dependent manner $(p<0.05$, Figure $1 \mathrm{~A})$. Increased $\mathrm{PGE}_{2}$ production by LPS was also significantly suppressed by $100 \mu \mathrm{g} / \mathrm{mL}$ EMM in RAW 264.7 cells (Figure 1B). To exclude the possibility that the decreased $\mathrm{NO}$ and $\mathrm{PGE}_{2}$ levels were due to cell death, cytotoxicity of EMM was determined by MTS assay. The result demonstrated that EMM showed no cytotoxicity in RAW 264.7 cells up to $100 \mu \mathrm{g} / \mathrm{mL}$ (Figure 1C). Thus, the inhibitory effects of EMM on NO and $\mathrm{PGE}_{2}$ production were not due to cytotoxicity.

\section{Effect of EMM on LPS-induced iNOS and COX-2 proteins and $\mathrm{mRNA}$ expression}

As shown in Figure 2, EMM strongly inhibited iNOS protein production in a dose-dependent manner; however, COX-2 protein production was only inhibited by $100 \mu \mathrm{g} / \mathrm{mL}$ EMM, which is similar pattern observed in the $\mathrm{PGE}_{2}$ secretion (Figure $1 \mathrm{~B}$ ). In addition to iNOS protein, EMM also inhibited iNOS mRNA expression in a dose-dependent manner. Like inhibition of COX-2 protein, COX-2 mRNA expression was partially inhibited by $100 \mu \mathrm{g} / \mathrm{mL}$ EMM. These results suggest that the EMM- mediated inhibition of $\mathrm{NO}$ and $\mathrm{PGE}_{2}$ production in LPS-stimulated macrophages is associated with downregulation of iNOS and COX-2 expression at transcriptional level.

\section{Effect of EMM on LPS-induced pro-inflammatory cytokines}

Increased levels of IL-1 $\beta$ (Figure 3A), IL-6 (Figure 3B), and TNF- $\alpha$ (Figure 3C) in RAW 264.7 cells by LPS stimulation were dramatically reduced in a dosedependent manner by exposure to EMM $(p<0.05)$. This result indicates that EMM efficiently suppressed LPSinduced IL-1 $\beta$, IL-6, and TNF- $\alpha$ release, which supports the hypothesis that EMM inhibits the initial phase of the LPS-stimulated inflammatory response.

\section{Effect of EMM on LPS-induced activation and translocation of NF-KB}

Confocal microscopy revealed that NF-kB/p65 was distributed mostly in the cytoplasm in unstimulated cells. After stimulation with LPS, most cytoplasmic NF- $\mathrm{BB} / \mathrm{p} 65$ were translocated into the nucleus, as shown by strong NF- $\mathrm{BB} / \mathrm{p} 65$ intensity in the nucleus (Figure 4A). The level of p65 in the nucleus was markedly reduced by treatment with EMM. Considering the inhibitory effects of EMM on LPS-induced NF- $\mathrm{kB}$ translocation into nucleus, we determined the effect of EMM on the promoter activity of NF-kB in LPS-stimulated cells. Data suggested that EMM treatment significantly inhibited LPS-induced NF- $\mathrm{kB}$ promoter activity (Figure $4 \mathrm{~B}, p<0.05$ ).

LPS treatment resulted in increased IKK $\beta$ phosphorylation and IkB- $\alpha$ degradation compared to non-treated

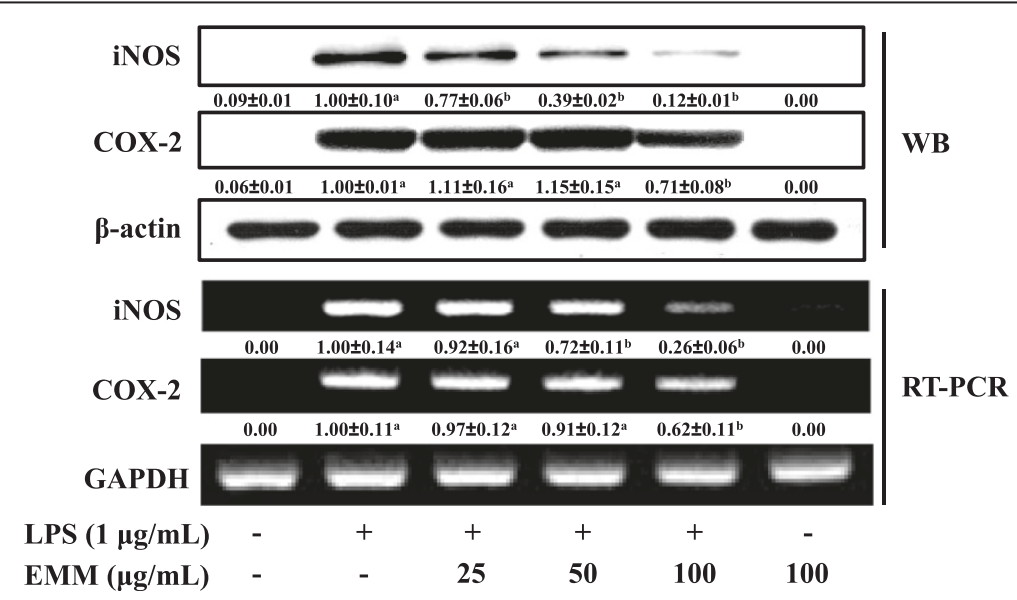

Figure 2 Effect of EMM on LPS-induced iNOS and COX-2 protein and mRNA expression in RAW 264.7 cells. (A) Cells were pretreated with indicated concentration of EMM for $1 \mathrm{~h}$ and stimulated with LPS $(1 \mathrm{\mu g} / \mathrm{mL})$ for $16 \mathrm{~h}$. Forty microgram of proteins were subjected to $10 \%$ SDS-PAGE. The expression of iNOS, COX-2, and $\beta$-actin protein was detected by Western blotting using corresponding antibodies. (B) Cells were pretreated with EMM for $1 \mathrm{~h}$ and stimulated with LPS for $6 \mathrm{~h}$, and then total RNA was prepared for RT-PCR. The results presented are representative of three independent experiments. ${ }^{a} p<0.05$ indicates significant differences compared to the control group. ${ }^{b} p<0.05$ indicates significant differences compared to the LPS-only treated group. 
(A)

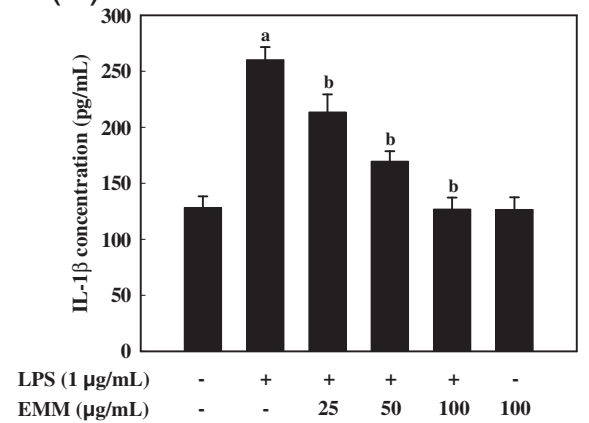

(C)

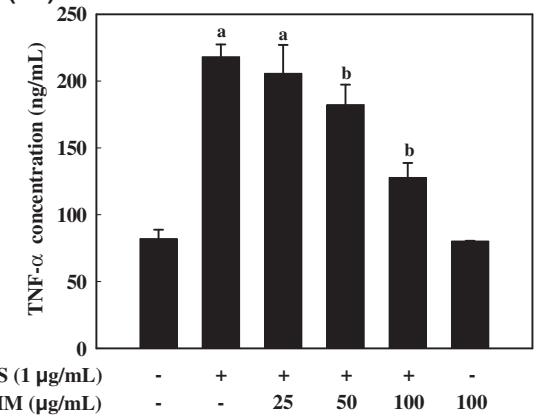

(B)

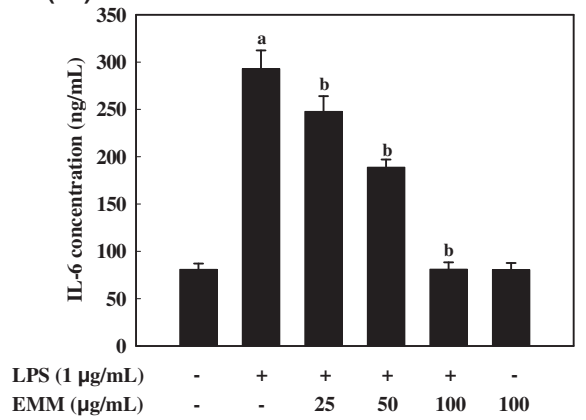

Figure 3 Effects of EMM on pro-inflammatory cytokine productions in LPS-stimulated RAW 264.7 cells. Cells were treated with various concentrations of EMM for $1 \mathrm{~h}$, and then stimulated with LPS for $24 \mathrm{~h}$. IL-1 $\beta(\mathbf{A}), \mathrm{LL}-6(\mathbf{B})$, and TNF-a (C) in the cultured media were measured by ELISA. Data are means \pm SDs of three independent experiments. ${ }^{a} p<0.05$ indicates significant differences compared to the control group. $\mathrm{b}_{p}<0.05$ indicates significant differences compared to the LPS-only treated group.

(A)

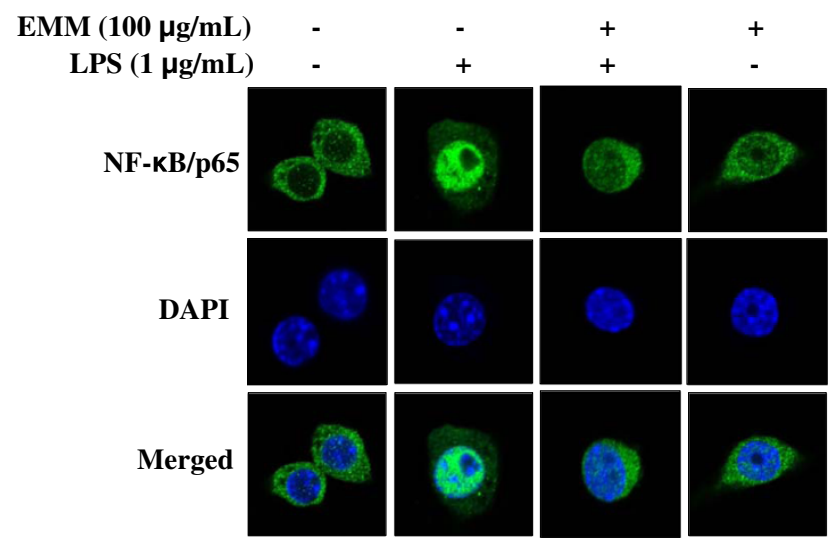

(B)

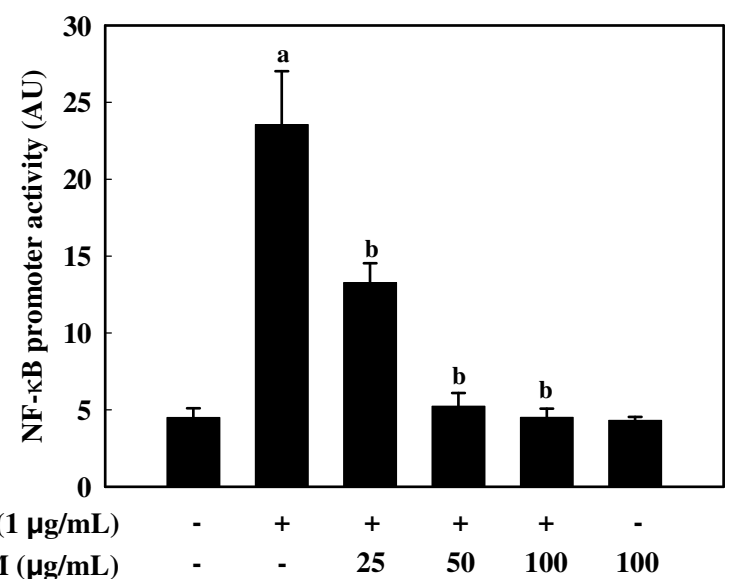

Figure 4 Effect of EMM on activation and translocation of NF-KB in RAW 264.7 cells. (A) Cells were pretreated with and without EMM for $1 \mathrm{~h}$ followed by LPS stimulation for 30 min. NF-KB/p65 subunits were probed by anti-NF-KB polyclonal primary antibody and Alexa Fluor ${ }^{\circledR}$ 488-conjugated secondary antibody. The nuclei were stained with DAPI and the images were captured by confocal microscopy. (B) Cells transfected with NF-KB promoter-containing luciferase DNA were pretreated with various concentrations of EMM for $1 \mathrm{~h}$ followed by LPS stimulation for 6 h. ${ }^{a} p<0.05$ indicates significant differences compared to the control group. ${ }^{b} p<0.05$ indicates significant differences compared to the LPS-only treated group. 


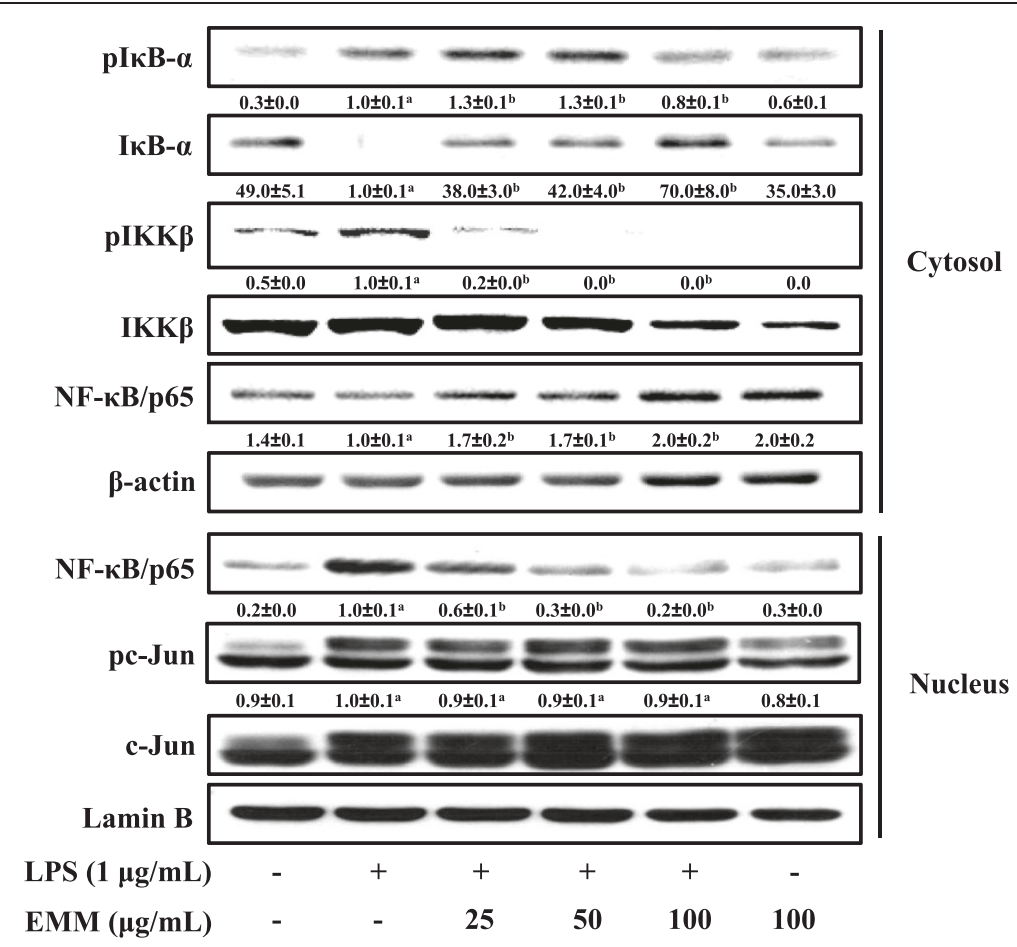

Figure 5 Inhibitory effect of EMM on the degradation of IKB- $\alpha$ and the activation of NF-KB in LPS-stimulated RAW 264.7 cells. Cells were incubated with various concentrations of EMM for $1 \mathrm{~h}$, and then stimulated with LPS $(1 \mu \mathrm{g} / \mathrm{mL})$ for 30 min. Cytosolic and nuclear fractions were prepared and analyzed by Western blotting using corresponding antibodies. The results presented are representative of three independent experiments. ${ }^{a} p<0.05$ indicates significant differences compared to the control group. ${ }^{b} p<0.05$ indicates significant differences compared to the LPS-only treated group.

control group, and EMM treatment suppressed IKK $\beta$ phosphorylation and ІкB- $\alpha$ degradation, recovered the control level of cytosolic IкB- $\alpha$ in a dose-dependent manner (Figure 5). As a result of IкB- $\alpha$ degradation, the increased NF- $\mathrm{KB}$ level in nucleus after LPS stimulation was reduced by EMM treatment in a dose-dependent manner (Figure 5). However, phosphorylation of c-Jun, a component of transcription factor AP-1, was not reduced by EMM treatment. These results indicate that the EMM-mediated inhibition of iNOS, COX-2, and pro-inflammatory cytokines production was mainly regulated by the transcription factor NF-кB in LPS-stimulated macrophages.

\section{Effect of EMM on LPS-induced phosphorylation of MAPKs and Akt}

As shown in Figure 6A, EMM inhibited phosphorylation of JNK, p38 MAPK, ERK, and Akt induced by LPS in RAW 264.7 cells, suggesting the additional characteristics of EMM to regulate NF- $\mathrm{KB}$ pathway via blocking the phosphorylation of MAPKs and Akt proteins in response to LPS signal. To further confirm the association of these signaling molecules with the EMM's anti-inflammatory effect, IкB- $\alpha$ phosphorylations in LPS-stimulated RAW 264.7 cells were measured in the presence of PI3K/Akt pathway inhibitor (Wortmannin), ERK inhibitor (PD98059), JNK inhibitor
(SP600125), or p38 inhibitor (SB203580). As shown in Figure $6 \mathrm{~B}, \mathrm{I} \mathrm{K} B-\alpha$ phosphorylation was remarkably suppressed by p38 inhibitor, which is consistent with the result of Figure 6A. Other inhibitors such as Wortmannin, PD98059, and SP600125, moderately inhibited the phosphorylation of IкB- $\alpha$. These data confirm additional characteristics of EMM on the phosphorylation of MAPKs and Akt proteins for NF- $\mathrm{KB}$ activation in response to LPS.

\section{Effect of EMM on PMA-induced ear edema in mouse}

Ear edema was induced by the application of PMA to the mouse ears. As shown in Figure 7, the application of PMA for $6 \mathrm{~h}$ significantly increased the weight of ear edema. Indo, a common clinical non-steroidal anti-inflammatory drug, was used as positive control for the inhibitory effect on the ear edema. As shown in Figure 7, pre-treatment of $1 \mathrm{mg} / \mathrm{ear}$ of Indo could effectively reduce ear edema after $6 \mathrm{~h}$ PMA stimulation $(p<0.05)$. Similarly, pre-administration of EMM $(90 \mu \mathrm{g} /$ ear $)$ markedly inhibited the PMA-induced ear edema with $66.8 \%$ inhibition $(p<0.05)$.

\section{Isolation of anti-inflammatory compounds from EMM}

EMM was further separated by reverse-phase column chromatography (Figure 8A) for the isolation of anti- 


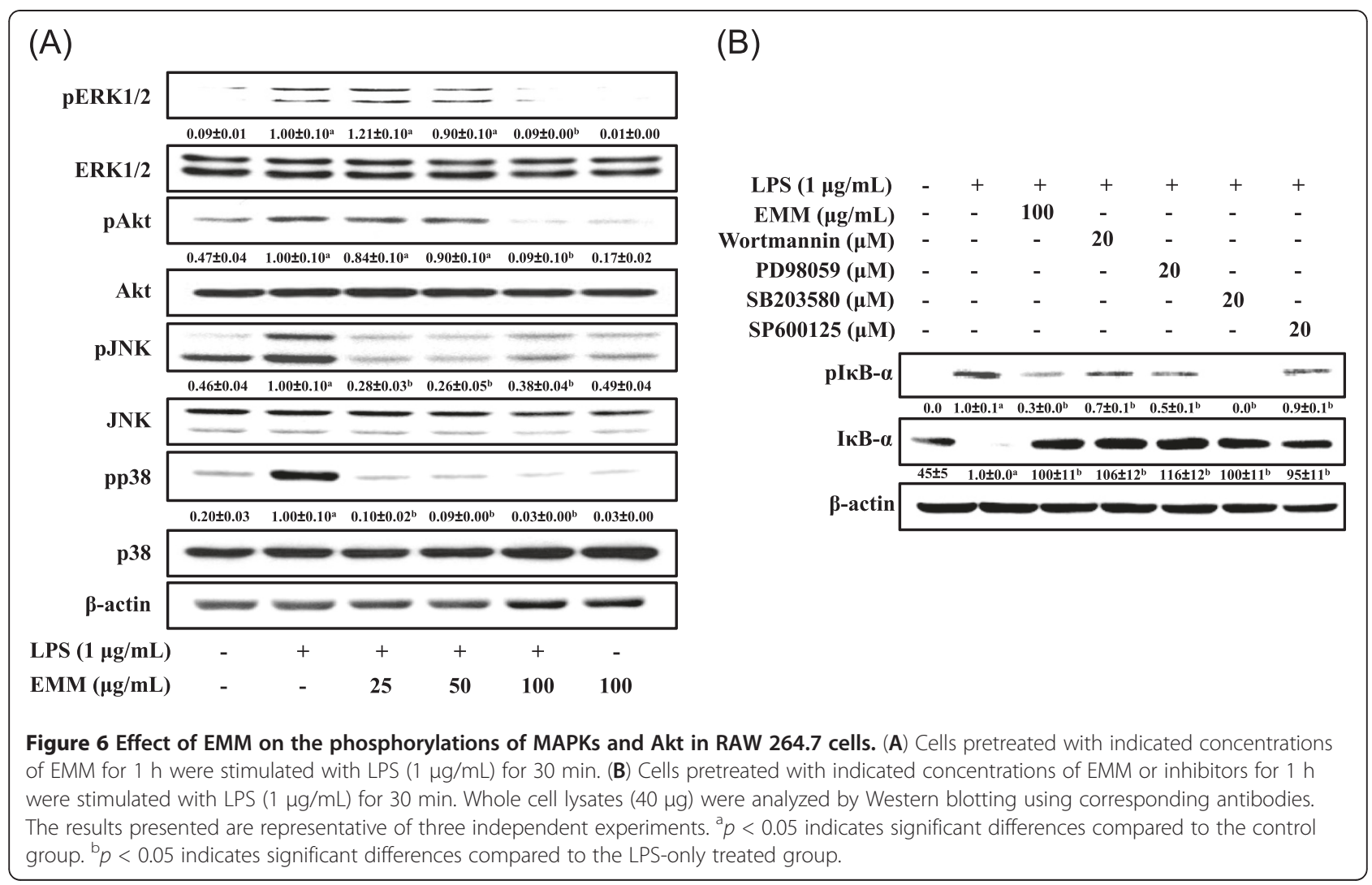

inflammatory compound(s). The chemical structures of the isolated compounds were identified as eckol and 6,6'bieckol, which were isolated for the first time from $M$.

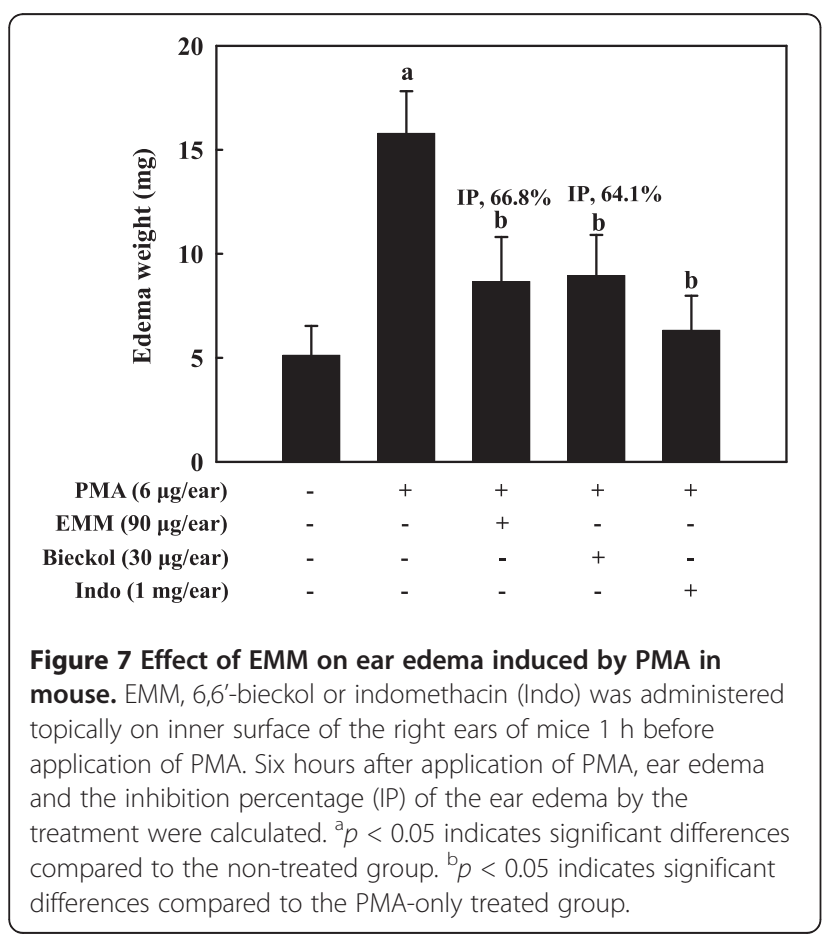

myagroides, from the comparison of their NMR spectra with the published spectral data. We obtained $2.5 \mathrm{mg}$ of eckol and $5.6 \mathrm{mg}$ of 6,6'-bieckol from $1 \mathrm{~g}$ of EMM. The purified 6,6'-bieckol significantly inhibited LPS-induced iNOS and COX-2 production in a dose-dependent manner $(p<0.05$, Figure 8B), however, eckol did not affect on the production of inflammatory proteins. Furthermore, preadministration of 6,6'-bieckol (30 $\mu \mathrm{g} /$ ear) markedly inhibited the PMA-induced ear edema with $64.1 \%$ inhibition (Figure 7, $p<0.05$ ). The result indicates that 6,6'-bieckol is one of the anti-inflammatory compounds in EMM.

\section{Discussion}

The present study was undertaken to examine the antiinflammatory effect of EMM on LPS-stimulated murine macrophage cells. To further understood the molecular mechanisms of EMM, we investigated the effects of EMM on the secretion of NO, PGE ${ }_{2}$, TNF- $\alpha$, IL- $1 \beta$, and IL-6, the expression of iNOS and COX-2, and the activation of NF- $\mathrm{kB}$. Our results indicated that EMM effectively inhibited the secretion of NO, PGE 2 , TNF- $\alpha$, IL- $1 \beta$, and IL- 6 through a blockade of the NF- $\mathrm{KB}$ and MAPK pathways in LPS-stimulated macrophages. The inhibitory effect of EMM on the expression of inflammatory mediators suggested one of the mechanisms responsible for its anti-inflammatory action and its potential for use as a therapeutic agent for treating inflammatory diseases. 

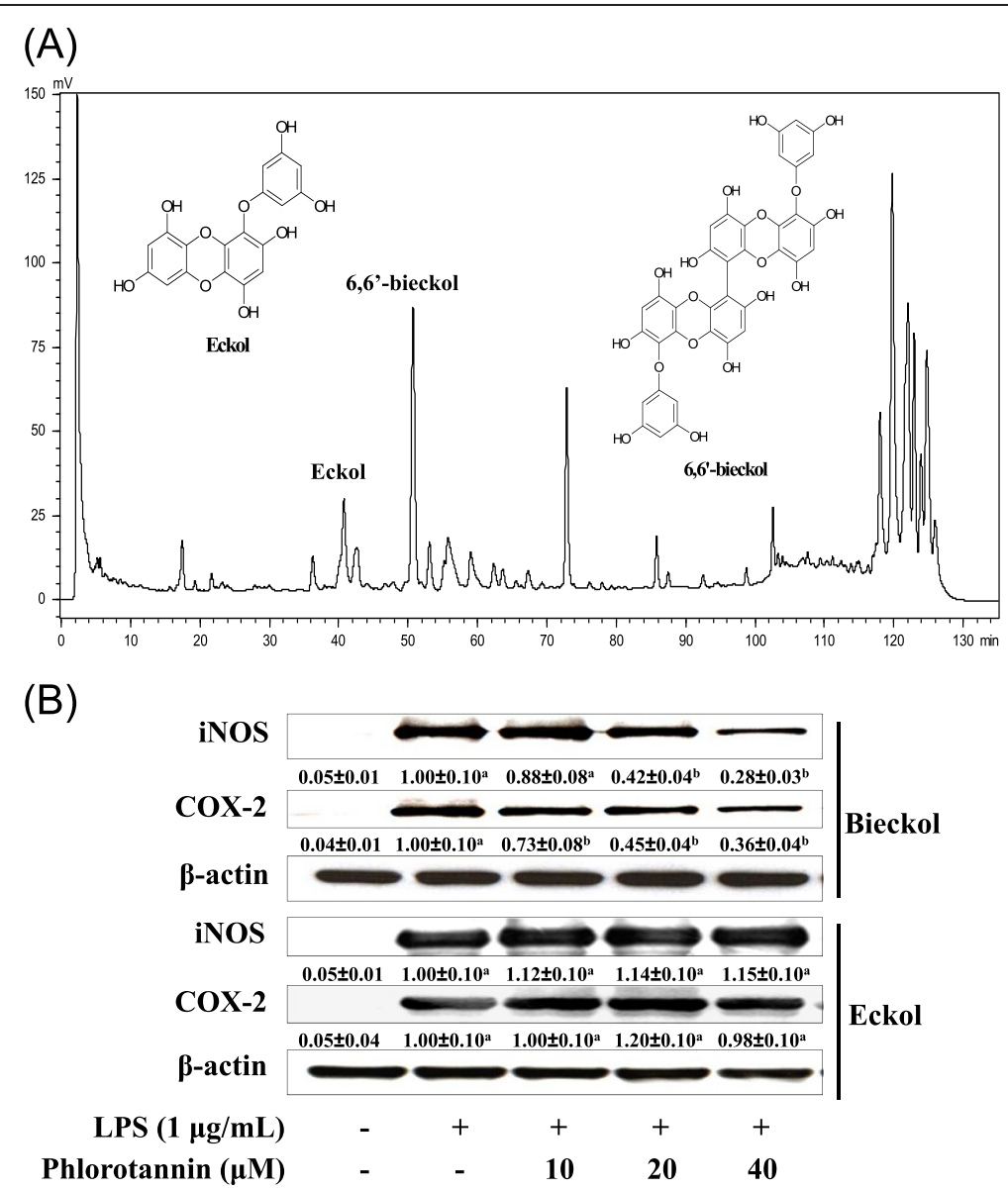

Figure 8 Identification of eckol and 6,6'-bieckol in EMM. (A) HPLC chromatogram of EMM and the chemical structures of eckol and 6,6'bieckol (insert). (B) Cells pretreated with different concentrations of eckol or 6,6'-bieckol for $1 \mathrm{~h}$ were stimulated with LPS for $16 \mathrm{~h}$. The expression levels of iNOS and COX-2 in cells were analyzed by Western blotting using corresponding antibodies. Relative density ratios of each protein over $\beta$-actin are shown below the blots. The results presented are representative of three independent experiments. ${ }^{a} p<0.05$ indicates significant differences compared to the control group. ${ }^{b} p<0.05$ indicates significant differences compared to the LPS-only treated group.

Under pathological conditions, excessive inflammatory mediators and pro-inflammatory cytokines produced by activated macrophages cause inflammatory process and act synergistically with other inflammatory mediators $[2,4,18]$. Compounds able to reduce $\mathrm{NO}$ or $\mathrm{PGE}_{2}$ production may be attractive as anti-inflammatory agents and, for this reason, the inhibitory effects of natural compounds on $\mathrm{NO}$ or $\mathrm{PGE}_{2}$ productions have been rigorously studied to develop therapeutic agent against inflammatory diseases $[17,19,20]$. Also, excessive production of pro-inflammatory cytokines plays a critical role in acute inflammatory responses as well as chronic inflammatory diseases. Recent studies have shown that in vivo or in vitro treatments of natural compounds are effective in reducing inflammation by the suppression of pro-inflammatory cytokines, which may ameliorate inflammation-related diseases, including atherosclerosis, cancer, and inflammatory arthritis [21,22]. Thus, the regulation of those molecules is important to the inhibition of inflammatory response. Our results indicate that the inhibition of $\mathrm{NO}$ and $\mathrm{PGE}_{2}$ production by EMM in LPS-stimulated macrophage cells is associated with downregulation of iNOS and COX-2 genes (Figure 2), which seems the first addressing transcriptional inhibition of iNOS and COX-2 by EMM.

$\mathrm{NF}-\mathrm{\kappa B}$ is a transcription factor actively involved in the transcriptional induction of iNOS and COX-2 gene $[1,3]$. Like NO and $\mathrm{PGE}_{2}$ produced by iNOS and COX-2, respectively, the release of pro-inflammatory cytokines is regulated by NF-kB pathway and plays an important role in the induction of the innate immune response of the acquired immune response [23]. It has been well studied that the inhibition of NF- $\mathrm{KB}$ activation by black tea extract is associated with the phosphorylation, ubiquitination, and subsequent degradation of ІкB via ubiquitin-proteosome pathway [24]. Although biochemical actions of EMM on NF- $\mathrm{kB}$ regulations remain unknown, the present study showed that 
EMM potentially inhibits the proteolytic degradation of I $\mathrm{B}-\alpha$ and the NF- $\kappa \mathrm{B}$ promoter-driven luciferase expression induced by LPS in RAW 264.7 cells. Therefore, these results demonstrate the ability of EMM to inhibit NF-kB activation in response to LPS signal in RAW 264.7 cells. Hence it is likely that reductions of iNOS, COX-2, and proinflammatory cytokine expression in RAW 264.7 cells are largely associated with activation/deactivation of NF-kB pathway. This report is, to our best knowledge, the novel findings to address the functions of EMM through NF-kB pathway in response to LPS treatment.

$\mathrm{NF}-\mathrm{kB}$ is also regulated by various signaling kinases including MAPKs (ERK, JNK, and p38) and Akt, which are groups of signaling molecules to play key roles in NF-kB activation [2,3]. MAPKs have been suggested to be involved in pro-inflammatory signaling cascades and in the activation of NF-kB in LPS-stimulated immune cells $[2,11]$. Therefore, anti-inflammatory mechanisms are closely related with inhibition of MAPKs in activated RAW 264.7 cells. In this study, we found that phosphorylation of MAPKs in response to LPS was inhibited by EMM treatment (Figure 6A). Interesting finding of this study is that the activation of Akt, a downstream regulator of PI3K, was also inhibited by EMM in response to LPS signal in RAW 264.7 cells. Precise mechanisms by which EMM modulate this PI3K/Akt pathway in the LPS stimulation remains unclear. Considering a recent study with a phlorofucofuroeckol A, which indicated a link between ROS and PI3K/Akt pathway in regulations of inflammatory genes $[7,11]$, it is speculated that the antioxidant activity of EMM or unknown components in EMM may be related to inhibition of Akt phosphorylation. Thus, it is likely that inhibition of MAPKs and Akt phosphorylation by EMM may contribute to the EMM-mediated inhibition of NF-kB pathway in LPS-stimulated RAW 264.7 cells.

Topical application of PMA to mouse skin induces inflammatory response and this is a well-established in vivo model for the evaluation of various anti-inflammatory agents. PMA can activate a wide variety of cell types that may contribute to acute inflammation, resulting in an increase in epidermal tissue swelling and infiltration of inflammatory cells $[25,26]$. The results from our in vivo experiments clearly showed that EMM $(90 \mu \mathrm{g} / \mathrm{ear})$ suppressed $66.8 \%$ of PMA-induced skin swelling. Furthermore, 6,6'-bieckol (30 $\mathrm{\mu g} / \mathrm{ear}$ ) isolated firstly from $M$. myagroides in this study inhibited $64.1 \%$ of PMA-induced skin swelling, indicating that it might be a main antiinflammatory compound in EMM. Although the inhibitory activity of EMM is about one third of 6,6'-bieckol, EMM showed higher anti-edema activity than Indo compared to its concentration. This result demonstrates that EMM can be used as a topical anti-inflammatory agent.

Like other polyphenolic compounds, phlorotannins, a class of compounds polymerized with phloroglucinol units found in brown seaweeds, have strong antioxidant [18,27] and anti-inflammatory $[11,28]$ activities. The multifunctional antioxidant activity of phlorotannins is highly associated with phenol rings which act as electron traps to scavenge peroxy, superoxide-anions, and hydroxyl radicals [29]. Specific mechanisms of phlorotannins on the antiinflammatory actions are not clearly defined. Considering cellular signaling of polyphenols, phlorotannins may not merely exert their effects as free radical scavenger, but may also modulate inflammatory cellular signaling proteins, including NF- $\mathrm{kB}$ and $\mathrm{AP}-1$ transcription factors [30]. Recent studies demonstrated that phlorofucofuroeckol A and 6,6'-bieckol isolated from Ecklonia spp. alleviate inflammatory response to LPS by inhibiting NF-kB pathway with its intrinsic antioxidant activity $[11,28]$. In the present study, the isolated 6,6'-bieckol from EMM remarkably inhibited the production of iNOS and COX-2 proteins, indicating 6,6'-bieckol is one of the principle anti-inflammatory compounds in EMM.

\section{Conclusions}

We demonstrated that EMM inhibited the secretion of inflammatory mediators, such as $\mathrm{NO}$ and $\mathrm{PGE}_{2}$, and pro-inflammatory cytokines, including TNF- $\alpha$, IL-1 $\beta$, and IL-6, in LPS-stimulated RAW 264.7 macrophages. Moreover, the inhibitory effect of EMM was associated with inactivation of the NF-kB pathway via blocking the phosphorylation of MAPKs and Akt. In addition, the results of in vivo study showed that EMM can be applicable to a topical anti-inflammatory agent.

\section{Abbreviations}

COX-2: Cyclooxygenase 2; DAPI: 4',6-Diamino-2-phenylindole; DMEM: Dulbecco's modified Eagle's medium; DMSO: Dimethyl sulfoxide; EMM: Ethanolic extract of Myagropsis myagroides; ERK: Extracellular signal regulated kinase; ELISA: Enzyme-linked immunosorbent assay; GAPDH: Glyceraldehyde 3-phosphate dehydrogenase; HPLC: Highperformance liquid chromatography; IKB: Inhibitor of KB; IKK: IkappaB kinase; IL: Interleukin; iNOS: Inducible nitric oxide synthase; JNK: c-Jun N-terminal kinase; LPS: Lipopolysaccharide; MAPK: Mitogen-activated protein kinase; NMR: Nuclear magnetic resonance; NF-kB: Nuclear factor-kB; NO: Nitric oxide; PBS: Phosphate-buffered saline; $\mathrm{PGE}_{2}$ : Prostaglandin $\mathrm{E}_{2}$;

PMSF: Phenylmethylsulfonyl fluoride; PCR: Polymerase chain reaction; TNF-a: Tumor necrosis factor-a; PMA: Phorbol 12-myristate 13-acetate.

\section{Competing interests}

The authors declare that they have no competing interests.

\section{Authors' contributions}

E-JJ carried out the main experiment and wrote the manuscript. M-SL prepared EMM and performed partial Western blotting. J-WC performed immunocytochemistry. JSK performed ELISA for measuring the proinflammatory cytokines. TS and B-MJ isolated and identified antiinflammatory compounds from EMM. NYY and C-WL harvested M. myogroides. J-IK contributed to discuss and write the manuscript. H-RK designed and organized this study. All authors read and approved the final version of the manuscript.

\section{Acknowledgements}

This work was financially supported by the National Fisheries Research and Development Institute (RP-2012-FS-015) and Brain Busan 21. 


\section{Author details}

${ }^{1}$ Department of Food Science and Nutrition, Pukyong National University, Busan 608-737, South Korea. ${ }^{2}$ Department of Food Science and Nutrition, Chonnam National University, Yeosu 550-749, South Korea. ${ }^{3}$ Food and Safety Research Division, National Fisheries Research and Development Institute, Gijang-gun, Busan 619-705, Korea.

Received: 21 May 2012 Accepted: 27 September 2012

Published: 3 October 2012

\section{References}

1. Xie QW, Whisnant R, Nathan C: Promoter of the mouse gene encoding calcium-independent nitric oxide synthase confers inducibility by interferon $\gamma$ and bacterial lipopolysaccharide. J Exp Med 1993, 177:1779-1784.

2. Zhang G, Ghosh S: Molecular mechanisms of NF-KB activation induced by bacterial lipopolysaccharide through Toll-like receptors. J Endotoxin Res 2000, 6:453-457.

3. Marks-Konczalik J, Chu SC, Moss J: Cytokine-mediated transcriptional induction of the human inducible nitric oxide synthase gene requires both activator protein 1 and nuclear factor kB-binding sites. J Biol Chem 1998, 273:22201-22208.

4. Vane JR, Mitchell JA, Appleton I, TomLinson A, Bishop-Bailey D, Croxtall J, Willoughby DA: Inducible isoforms of cyclooxygenase and nitric-oxide synthase in inflammation. Proc Natl Acad Sci U S A 1994, 91:2046-2050.

5. Makarov SS: NF-KB in rheumatoid arthritis: a pivotal regulator of inflammation, hyperplasia, and tissue destruction. Arthritis Res Ther 2001, 3:200-206.

6. Guha M, Mackman N: LPS induction of gene expression in human monocytes. Cell Signal 2001, 13:85-94.

7. Kao SJ, Lei HC, Kuo CT, Chang MS, Chen BC, Chang YC, Chiu WT, Lin CH: Lipoteichoic acid induces nuclear factor- $\mathrm{KB}$ activation and nitric oxide synthase expression via phosphatidylinositol 3-kinase, Akt, and p38 MAPK in RAW 264.7 macrophages. Immunology 2005, 115:366-374.

8. Zou Y, Qian ZJ, Li Y, Kim MM, Lee SH, Kim SK: Antioxidant effects of phlorotannins isolated from Ishige okamurae in free radical mediated oxidative systems. J Agric Food Chem 2008, 56:7001-7009.

9. Okada Y, Ishimaru A, Suzuki R, Okuyama T: A new phloroglucinol derivative from the brown alga Eisenia bicyclis: potential for the effective treatment of diabetic complications. J Nat Prod 2004, 67:103-105.

10. Yoon NY, Chung HY, Kim HR, Choi JS: Acetyl- and butyrylcholinesterase inhibitory activities of sterols and phlorotannins from Ecklonia stolonifera. Fish Sci 2008, 74:200-207.

11. Kim AR, Lee MS, Shin TS, Hua H, Jang BC, Choi JS, Byun DS, Utsuki T, Ingram $D$, Kim HR: Phlorofucofuroeckol A inhibits the LPS-stimulated iNOS and COX-2 expressions in macrophages via inhibition of NF-KB, Akt, and p38 MAPK. Toxicol In Vitro 2011, 25:1789-1795

12. Lee MS, Shin TS, Utsuki T, Choi JS, Byun DS, Kim HR: Isolation and identification of phlorotannins from Ecklonia stolonifera with antioxidant and epatoprotective properties in tacrine-treated HepG2 cells. J Agric Food Chem 2012, 60:5340-5349.

13. Wong CK, Ooi VE, Ang PO: Protective effects of seaweeds against liver injury caused by carbon tetrachloride in rats. Chemosphere 2000 41:173-176.

14. Heo SJ, Yoon WJ, Kim KN, Ahn GN, Kang SM, Kang DH, Affan A, Oh C, Jung WK, Jeon YJ: Evaluation of anti-inflammatory effect of fucoxanthin isolated from brown algae in lipopolysaccharide-stimulated RAW 264.7 macrophages. Food Chem Toxicol 2010, 48:2045-2051.

15. Kim H, Lee HS, Chang KT, Ko TH, Baek KJ, Kwon NS: Chloromethyl ketones block induction of nitric oxide synthase in murine macrophages by preventing activation of nuclear factor-KB. J Immunol 1995, 154:4741-4748.

16. Lee JY, Lee MS, Choi HJ, Choi JW, Shin T, Woo HC, Kim Jl, Kim HR: Hexane fraction from Laminaria japonica exerts anti-inflammatory effects on lipopolysaccharide-stimulated RAW 264.7 macrophages via inhibiting NF-kappaB pathway. Eur J Nutr 2012, [Epub ahead of print].

17. Kim AR, Shin TS, Lee MS, Park JY, Park KE, Yoon NY, Kim JS, Choi JS, Jang BC, Byun DS, Park NK, Kim HR: Isolation and identification of phlorotannins from Ecklonia stolonifera with antioxidant and anti-inflammatory properties. J Agric Food Chem 2009, 57:3483-3489.
18. Libby P: Inflammation and cardiovascular disease mechanisms. Am J Clin Nutr 2006, 83:456S-460S

19. Gupta M, Mazumder UK, Gomathi P, Selvan VT: Antiinflammatory evaluation of leaves of Plumeria acuminata. BMC Complement Altern Med 2006, 6:36

20. Elmann A, Mordechay S, Erlank H, Telerman A, Rindner M, Ofir R: Antineuroinflammatory effects of the extract of Achillea fragrantissima. BMC Complement Altern Med 2011, 11:98.

21. Garcia-Lafuente A, Guillamon E, Villares A, Rostagno MA, Martinez JA: Flavonoids as anti-inflammatory agents: implications in cancer and cardiovascular disease. Inflamm Res 2009, 58:537-552.

22. Ekambaram S, Perumal SS, Subramanian V: Evaluation of antiarthritic activity of Strychnos potatorum Linn seeds in Freund's adjuvant induced arthritic rat model. BMC Complement Altern Med 2010, 10:56.

23. Netea MG, Warris A, Van der Meer JW, Fenton MJ, Verver-Janssen TJ, Jacobs $L E$, Andresen T, Verweij PE, Kullberg BJ: Aspergillus fumigatus evades immune recognition during germination through loss of toll-like receptor-4-mediated signal transduction. J Infect Dis 2003, 188:320-326.

24. Song YA, Park YL, Kim KY, Chung CY, Lee GH, Cho DH, Ki HS, Park KJ, Cho SB, Lee WS, Kim N, Ahn BW, Joo YE: Black tea extract prevents lipopolysaccharide-induced NF-KB signaling and attenuates dextran sulfate sodium-induced experimental colitis. BMC Complement Altern Med 2011, 11:91.

25. Wershil BK, Murakami T, Galli SJ: Mast cell-dependent amplification of an immunologically nonspecific inflammatory response. Mast cells are required for the full expression of cutaneous acute inflammation induced by phorbol 12-myristate 13-acetate. J Immunol 1988, 140:2356-2360.

26. Garrido G, Gonzalez D, Lemus Y, Garcia D, Lodeiro L, Quintero G, Delporte C, Nunez-Selles AJ, Delgado R: In vivo and in vitro anti-inflammatory activity of Mangifera indica L. extract (VIMANGR ${ }^{\circledR}$ ). Pharmacol Res 2004, 50:143-149.

27. Kang HS, Chung HY, Kim JY, Son BW, Jung HA, Choi JS: Inhibitory phlorotannins from the edible brown alga Ecklonia stolonifera on total reactive oxygen species (ROS) generation. Arch Pharm Res 2004, 27:194-198.

28. Yang Yl, Shin HC, Kim SH, Park WY, Lee KT, Choi JH: 6,6'-Bieckol, isolated from marine alga Ecklonia cava, suppressed LPS-induced nitric oxide and $\mathrm{PGE}_{2}$ production and inflammatory cytokine expression in macrophages: The inhibition of NF-KB. Int Immunopharmacol 2012, 12:510-517.

29. Wang T, Jónsdóttir R, Ólafsdóttir G: Total phenolic compounds, radical scavenging and metal chelation of extracts from Icelandic seaweeds. Food Chem 2009, 116:240-248.

30. Rahman I, Biswas SK, Kirkham PA: Regulation of inflammation and redox signaling by dietary polyphenols. Biochem Pharmacol 2006, 72:1439-1452.

doi:10.1186/1472-6882-12-171

Cite this article as: Joung et al:: Anti-inflammatory effect of ethanolic extract from Myagropsis myagroides on murine macrophages and mouse ear edema. BMC Complementary and Alternative Medicine 2012 $12: 171$

\section{Submit your next manuscript to BioMed Central and take full advantage of:}

- Convenient online submission

- Thorough peer review

- No space constraints or color figure charges

- Immediate publication on acceptance

- Inclusion in PubMed, CAS, Scopus and Google Scholar

- Research which is freely available for redistribution 